\title{
ON A HIERARCHY OF GENERALIZED DIAGONAL DOMINANCE PROPERTIES FOR COMPLEX MATRICES ${ }^{1}$
}

\section{J. L. BRENNER AND W. G. BROWN}

Abstract. This article concerns dominance conditions for an $n \times m$ matrix. In the simplest kind of dominance, the (absolute) value of the diagonal element exceeds the sum of the absolute values of the nondiagonal elements on the same row. This condition has been generalized in the literature in several ways, of which we consider ways in which the rows of the matrix cooperate. Our work amounts to a sorting out of certain dominance conditions that belong to a class $\mathcal{C}$ of dominance conditions. We prove a theorem characterizing all true statements of the form

$$
C_{1}, C_{2}, \cdots, C_{8} \Rightarrow C_{0}
$$

where $C_{i} \in \mathcal{C}(i=0,1, \cdots, s)$.

1. Introduction. By way of orientation, we note the following. If the simplest kind of dominance obtains (i.e. if in any row the absolute value of the diagonal element exceeds the sum of the absolute values of the nondiagonal elements) the principal submatrix of an $n \times m$ matrix is necessarily nonsingular. Another sufficient condition for nonsingularity is the following: Let the rows of $A$ be divided into disjoint sets, and suppose that in each set of rows, and for each $t$, the absolute value of the determinant of the principal submatrix exceeds the sum of the absolute values of those nonprincipal submatrices that can be obtained by replacing the $t$ th (fixed) column of the principal matrix by another column (from the same set of rows). Then $A$ is nonsingular. There exist generalizations of the dominance idea to contexts in which the elements of $A$ are operators on assortments of vector spaces.

2. The class $\mathcal{C}$. $A$ will always be an $n \times m$ complex matrix $(n \leqq m)$. $A_{1}$ is the principal submatrix, i.e. the $n \times n$ submatrix of $A$ consisting of the first $n$ columns (and all $n$ rows on those columns).

$n$ denotes the set of the first $n$ natural numbers $(n>0)$. Let $X$ be some set of vectors in $C^{n}$, usually a set of columns of $A$, but in some

Received by the editors July 16, 1969.

AMS Subject Classifications. Primary 1558; Secondary 1555, 0525.

Key Words and Phrases. Matrix diagonal dominance, matrix nonsingularity criteria.

1 This research was supported in part by the National Research Council of Canada, and by the National Science Foundation under grant GP 9483. 
cases a set of indeterminate vectors. Let $A \subset n$, and let $\phi: \Lambda \rightarrow X$ be any one-to-one mapping, so that the image of $\phi$ is a set of $m$ vectors $(m \leqq n) . A(\phi)$ denotes the $n \times n$ matrix obtained from $A_{1}$ by replacing every column with index in $\Lambda$, the $\lambda$ th column being replaced by $\phi(\lambda)(\lambda \in \Lambda) . C_{\Lambda}(A)$ will denote the statement

$$
C_{\Lambda}(A):\left|\operatorname{det} A_{1}\right|>\frac{1}{(\operatorname{card} \Lambda) !} \sum_{\phi}|\operatorname{det} A(\phi)| ;
$$

where summation is effected over all one-to-one mappings $\phi$ from $\Lambda$ into the column set of $A \backslash A_{1}$. If the sum is empty, it is defined as 0 . By card $\Lambda$ we denote the cardinality of $\Lambda$. If we had chosen to require $\phi$ to be order-preserving (relative to any fixed order on $X$ ) we could restate $(2.1)$ without the factor $\{(\operatorname{card} \Lambda) !\}^{-1}$.

Several questions arise naturally in connection with a matrix, or the set of matrices, that satisfy some set of conditions (2.1). For example:

I. What are the logical relations between the statements $C_{\Lambda}(A)$ for $A$ fixed, but $\Lambda$ variable?

II. What relations can be asserted between the various statements $C_{\Lambda}(A)$ as $A$ ranges over the row sets of a given matrix?

III. What can be said about generalizations of $C_{\Lambda}(A)$, for example to the case where the right side is the sum of several partial sums, and in each partial sum a different set of columns of $A_{1}$ is held fixed?

ExAmples. Ordinary dominance $\left|a_{11}\right|>\sum_{j>1}\left|a_{1 j}\right|$ is the condition $C_{\Lambda}(A)$ in case $n=1$ and $\Lambda=(1)$. Two-row dominance, with the first column replaced, is given by $n=2$ and $\Lambda_{1}=\{1\}$. Two-row dominance with the second column replaced is the condition $\left(\Lambda_{2}=\{2\}\right)$

$$
C_{\Lambda_{2}}(A):\left|\operatorname{det} A\left(\begin{array}{c}
12 \\
12
\end{array}\right)\right|>\sum_{j>2}\left|\operatorname{det} A\left(\begin{array}{c}
12 \\
1 j
\end{array}\right)\right| \text {. }
$$

Two-row dominance with both columns replaced is the condition $(\Lambda=2)$

$$
C_{2}(A):\left|\operatorname{det} A\left(\begin{array}{c}
12 \\
12
\end{array}\right)\right|>\sum_{i>j>2}\left|\operatorname{det} A\left(\begin{array}{c}
12 \\
i j
\end{array}\right)\right| .
$$

We show below that this condition is implied by the conjunction of the two preceding ones.

In this paper we shall consider only questions of type I. Questions of types II and III have been studied in [1]; the methods used there even apply to questions (parallel to (2.1)) on permanents.

For any subset $\Lambda_{i}$ of $n, \chi_{i}$ will denote the characteristic function defined by 


$$
\begin{aligned}
\chi_{i}(p) & =1, & & p \in \Lambda_{i}, \\
& =0, & & p \in \Lambda_{i} .
\end{aligned}
$$

Our main result is the following

(2.2) Theorem. Let $\Lambda_{i} \in n(i=0,1, \cdots, s>0)$. If the validity of all conditions $C_{\Lambda_{i}}$ (conjunction) implies condition $C_{\Lambda_{0}}$, then there exist nonnegative reals $r_{0}, r_{1}, \cdots, r_{s}$, not all zero, such that

$$
\sum_{i=1}^{s} r_{i} \chi_{i}=r_{0} \chi_{0} \text {. }
$$

Conversely, if (2.3) holds, then $C_{\Lambda_{i}}$ in their collection imply $C_{\Lambda_{0}}$. Compactly,

$$
\begin{aligned}
{\left[\begin{array}{c}
\left\{\begin{array}{c}
s \\
\Lambda_{i} \\
1
\end{array} C_{\Lambda_{i}}\right\} \rightarrow C_{\Lambda_{0}}
\end{array}\right] \leftrightarrow \exists\left\{r_{i}\right\} \mid\left(\left\{r_{i}\right\}\right.} & \left.\neq\left\{0_{i}\right\}\right) \\
& \wedge\left(\bigwedge_{i}\left(r_{i} \geqq 0\right)\right) \wedge\left(\sum_{1}^{s} r_{i} \chi_{i}=r_{0} \chi_{0}\right) .
\end{aligned}
$$

3. Proof of necessity $(\rightarrow)$. Let $\Lambda_{0}, \Lambda_{1}, \cdots, \Lambda_{s}$ be subsets of $n$ for which there exist no nonnegative $r_{0}, r_{1}, \cdots, r_{s}$ satisfying (2.3). We shall prove the existence of a matrix $A$ for which all $C_{\Lambda_{i}}$ hold, but $C_{\Lambda_{0}}$ does not, providing only that $m \geqq n+$ card $\Lambda_{0}$. The matrix $A$ whose existence we shall demonstrate (our proof is not constructive) will be formed by bordering (augmenting) the $n \times n$ identity matrix on the right with the real diagonal matrix $\operatorname{diag}\left(x_{1}, x_{2}, \ldots\right)$. For such a matrix, that $A$ has the property $C_{\Lambda_{i}}$ means that $1>\prod_{j \in \Lambda_{i}}\left|x_{j}\right|$. Thus we wish to solve simultaneously the inequalities

$$
\begin{aligned}
& \prod_{j \in \Lambda_{i}}\left|x_{j}\right|<1 \quad(i=1, \cdots, s), \\
& \prod_{j \in \Lambda_{0}}\left|x_{j}\right| \geqq 1 .
\end{aligned}
$$

We consider, more conveniently, the corresponding equations in $y_{j}=\log \left|x_{j}\right|$ :

$$
\begin{aligned}
& \sum_{j \in \Lambda_{i}} y_{j}<0 \quad(i=1, \cdots, s), \\
& \sum_{j \in \Lambda_{0}} y_{j} \geqq 0 .
\end{aligned}
$$

We shall prove the existence of a solution to (3.2), even with strict inequality in the last statement. 
For this purpose we shall require the following somewhat more general result, which we state without proof.

(3.3) Lemma. Let $V$ be a finite-dimensional real vector space; let $\Phi$, $\Psi$ be finite subsets of the dual space $V^{*}$. Then the system of inequalities

$$
f x<0 \text { for all } f \in \Phi, \quad g x>0 \text { for all } g \in \Psi
$$

has a solution $x \in V$ if and only if

$$
\begin{aligned}
& \sum r_{f} f=\sum s_{o} g, \text { with } r_{f} \text { and } s_{o} \text { nonnegative scalars, almost all } \\
& \text { zero, implies that } r_{f}=0 \text { for all } f \in \Phi, \text { and } s_{o}=0 \text { for all } g \in \Psi .
\end{aligned}
$$

(We remark that (3.3) is true without the hypothesis of finiteness for $\Phi$ and $\Psi$. A proof can be based on Theorem (2.7) of [2].)

We return now to the system (4.2) which we wish to solve. We define the functional $f_{i}$ by

$$
f_{i}\left(y_{1}, \cdots, y_{n}\right)=\sum_{j} \chi_{i}(j) y_{j} \quad(i=0,1, \cdots, s) .
$$

System (3.2) can be rewritten

$$
f_{i} y<0 \quad(i=1, \cdots, s), \quad f_{0} y \geqq 0 .
$$

By hypothesis, there do not exist nonnegative integers $r_{0}, r_{1}, \cdots, r_{\text {c }}$ not all zero, such that

$$
\sum_{i=1}^{s} r_{i} f_{i}=r_{0} f_{0}
$$

But now conditions (3.3) are satisfied. Thus there exists a solution to (3.6) and hence to (3.2). This completes the proof of necessity.

4. Proof of sufficiency $(\leftarrow)$. We assume $\Lambda_{i} \subset n(i=0,1, \cdots, s>0)$ are subsets and $r_{0}, r_{1}, \cdots, r_{s}$ are nonnegative integers, not all zero, such that (2.3) holds. As the functions $\chi_{i}$ take integer values on $\boldsymbol{n}$ the existence of a real solution $r_{0}, r_{1}, \cdots, r_{s}$ of (3.3) implies the existence of a solution in integers; we may thus assume $r_{0}, r_{1}, \cdots, r_{s}$ to be integers. Since there is no restriction that $\Lambda_{i}$ be distinct, we can assume, without limiting generality, that $r_{i}=1(i=1, \cdots, s)$.

We define $t_{i}=$ card $\Lambda_{i}, i=1, \cdots, s$. Then (2.3) with $r_{i}=1$ for $i>0$, implies that $\sum_{i=1}^{s}\left(t_{0}-t_{i}\right)=s t_{0}-\sum_{i=1}^{s} t_{i}=\left(s-r_{0}\right) t_{0}$. Now let $Y_{1}, \cdots$, $Y_{s-r_{0}}$ each be a set of $t_{0}$ column matrices of size $n \times 1$, whose entries are $n t_{0}\left(s-r_{0}\right)$ distinct indeterminates. Define $X=\bigcup_{h=1}^{s-r_{0}} Y_{h}$. Let $\phi_{i}: \Lambda_{i} \rightarrow X$ $(i=1, \cdots, s)$ be any set of one-to-one mappings whose images partition $X$; and let $\psi_{j}: \Lambda_{0} \rightarrow X$ be any one-to-one mapping whose image is $Y_{j}\left(j=1, \cdots, s-r_{0}\right)$. 
(4.1) Lemma. $\sum_{1} \prod_{i=1}^{s} \operatorname{det} A\left(\phi_{i}\right)=\left(\operatorname{det} A_{1}\right)^{r_{0}} \prod_{h=1}^{s-r_{0}} \operatorname{det} A\left(\psi_{j}\right)$, where the summation $\sum_{1}$ is extended over all determinantal permutations of each set $Y_{h}\left(h=1, \cdots, s-r_{0}\right)$.

Proof. This follows from a theorem of Turnbull [3, pp. 48-49], applied $s-r_{0}$ times.

(4.2) Corollary 1.

$$
\sum_{1} \prod_{i=1}^{s}\left|\operatorname{det} A\left(\phi_{i}\right)\right| \geqq\left|\operatorname{det} A_{1}\right| \text { ro }^{8} \prod_{j=1}^{8-r_{0}}\left|\operatorname{det} A\left(\psi_{j}\right)\right| .
$$

(4.3) Corollary 2. Suppose each of the indeterminate column vectors from $X$ is allowed to range over a set $W$ of vectors of $C^{n}$. Then if $\sum_{2}$ denotes summation over this range, we find from (4.2)

$$
\sum_{2} \sum_{1} \prod_{i=1}^{s}\left|\operatorname{det} A\left(\phi_{i}\right)\right| \geqq\left|\operatorname{det} A_{1}\right|^{r_{0}} \sum_{2} \prod_{j=1}^{s-r_{0}}\left|\operatorname{det} A\left(\psi_{\jmath}\right)\right|
$$

i.e.

$$
\begin{aligned}
\frac{\left(t_{0} !\right)^{s-r_{0}}}{t_{1} ! \cdots t_{s} !} & \sum_{2} \prod_{i=1}^{s}\left|\operatorname{det} A\left(\phi_{i}\right)\right| \\
& \geqq\left|\operatorname{det} A_{1}\right|^{r_{0}}\left(\sum|\operatorname{det} A(\phi)|\right)^{s-r_{0}}
\end{aligned}
$$

where $\sum$ is effected over all one-to-one mappings $\phi: \Lambda_{0} \rightarrow W$.

We now complete the proof of sufficiency. We are assuming $C_{\Lambda_{i}}(A)$ is true $(i=1, \cdots, s)$. The product of these $s$ inequalities $(2.1)$ is

$$
\begin{aligned}
\left|\operatorname{det} A_{1}\right|^{s} & >\frac{1}{t_{1} ! \cdots t_{s} !} \prod \sum_{\phi_{i}}\left|\operatorname{det} A\left(\phi_{i}\right)\right| \\
& =\frac{1}{t_{1} ! \cdots t_{s} !} \sum_{2} \prod_{i=1}^{s}\left|\operatorname{det} A\left(\phi_{i}\right)\right| .
\end{aligned}
$$

But by (4.3), this is no less than

$$
\left(t_{0} !\right)^{-s+r_{0}}\left|\operatorname{det} A_{1}\right|^{r_{0}}\left(\sum_{\phi}|\operatorname{det} A(\phi)|\right)^{s-r_{0}}
$$

where $\sum_{\phi_{i}}$ and $\sum_{\phi}$ are effected over all one-to-one mappings $\phi_{i}: \Lambda_{i} \rightarrow W$ and $\phi: \Lambda_{0} \rightarrow W$ respectively, $W$ being the column set of $A \backslash A_{1}$. Any of the statements $C_{\Lambda_{i}}(A)$ implies that $A_{1}$ is nonsingular. Hence (4.5) may be rewritten as 


$$
\left|\operatorname{det} A_{1}\right|^{\circ-r_{0}}>\left(\frac{1}{t_{0} !} \sum_{\phi}|\operatorname{det} A(\phi)|\right)^{s-r_{0}},
$$

which is merely a paraphrase of $C_{\Lambda_{0}}(A)$.

5. Further questions. With fixed $s, 2<s \leqq m$, the condition

$$
\left|\operatorname{det} A\left(\begin{array}{l}
12 \\
12
\end{array}\right)\right|>\sum_{2<j \leq s}\left|\operatorname{det} A\left(\begin{array}{c}
12 \\
1 j
\end{array}\right)\right|+\sum_{i>j>\mathbf{s}}\left|\operatorname{det} A\left(\begin{array}{c}
12 \\
i j
\end{array}\right)\right|
$$

is a consequence of the two conditions $C_{\Lambda_{i}}, C_{\Lambda_{2}}$ in the examples at the end of $\S 2$. Moreover, the conjunction of (5.1) and $C_{\Lambda_{2}}$ imply $C_{2}$ of $\S 2$.

The inequalities can all be made more delicate if the bare inequality sign is replaced by a refined estimate of the ratio of the two members of the inequality. Thus if the left members of $C_{\Lambda_{1}}, C_{\Lambda_{2}}$ are provided with additional positive factors $\sigma_{1}, \sigma_{2}$ respectively, $0<\sigma_{1}, \sigma_{2}<1$, their conjunction implies the statement

$$
\sigma_{1} \sigma_{2}\left|\operatorname{det} A\left(\begin{array}{l}
12 \\
12
\end{array}\right)\right|>\sum_{i>j>2}\left|\operatorname{det} A\left(\begin{array}{l}
12 \\
12
\end{array}\right)\right|,
$$

which is stronger than $C_{2}$ of $\S 2$.

\section{REFERENCES}

1. J. L. Brenner, Relations among the minors of a matrix with dominant principal diagonal, Duke Math. J. 26 (1959), 563-567. MR 22 \#1590.

2. V. Klee, Jr., Separation properties of convex cones, Proc. Amer. Math. Soc. 6 (1955), 313-318. MR 16, 832.

3. H. W. Turnbull, The theory of determinants, matrices, and invariants, 3rd ed., Dover, New York, 1960. MR 24 \#A123.

University of Arizona, Tucson, Arizona 85721

McGill University, Montreal 110, Quebec, Canada 